\title{
Verbal and nonverbal intelligence changes in the teenage brain
}

\author{
Sue Ramsden ${ }^{1}$, Fiona M. Richardson ${ }^{1}$, Goulven Josse ${ }^{1}$, Michael S. C. Thomas ${ }^{2}$, Caroline \\ Ellis ${ }^{1}$, Clare Shakeshaft ${ }^{1}$, Mohamed L. Seghier ${ }^{1}$, and Cathy J. Price ${ }^{1}$ \\ ${ }^{1}$ Wellcome Trust Centre for Neuroimaging, UCL, London WC1N 3BG, UK \\ ${ }^{2}$ Developmental Neurocognition Laboratory, Department of Psychological Sciences, Birkbeck \\ College, University of London WC1E 7HX
}

\begin{abstract}
Intelligence Quotient (IQ) is a standardized measure of intellectual ability that taps a wide range of cognitive skills ${ }^{1}$. Across life span, IQ is generally considered to be stable with scores at one time point used to predict educational achievement and employment prospects in later years ${ }^{1}$. Neuroimaging allows us to test whether unexpected longitudinal fluctuations in measured IQ are related to brain development. Here we show that verbal and nonverbal IQ can rise or fall in the teenage years, with these changes in performance validated by their close correlation with changes in local brain structure. A combination of structural and functional imaging showed that verbal IQ changed with grey matter in an area that was activated by speech, while nonverbal IQ changed with grey matter in an area that was activated by finger movements. By using longitudinal assessments of the same individuals, we eschewed the many sources of variation in brain structure that confound cross sectional studies. This allowed us to dissociate neural markers for verbal and nonverbal IQ and to show that these general abilities are closely linked to the sensorimotor skills involved in learning. More generally, our results emphasize the possibility that an individual's intellectual capacity relative to their peers can weaken or strengthen in the teenage years. This would be encouraging to those whose intellectual potential may improve; and a warning that early achievers may not maintain their potential.
\end{abstract}

\begin{abstract}
An individual's abilities and capacity to learn can be partially captured by the use of verbal and nonverbal intelligence tests. The Intelligence Quotient (IQ) provides a standardized method for measuring intellectual abilities and is widely used within education, employment and clinical practice. In the absence of neurological insult or degenerative conditions, IQ is usually expected to be stable across lifespan, as evidenced by the fact that IQ measurements taken at different points in an individual's life tend to correlate well ${ }^{1-2}$. Nevertheless, strong correlations over time disguise considerable individual variation: for example a correlation coefficient of .7 (which is not unusual with verbal IQ) still leaves over $50 \%$ of the variation unexplained. The current study tested whether variation in a teenager's IQ over time correlated with changes in brain structure. This would provide construct validity for the growth or decline of IQ in the teenage years because if IQ changes correspond to structural brain changes, then they are unlikely to represent measurement error in the IQ tests. In addition, if verbal and nonverbal skills change at different rates in different individuals, the
\end{abstract}

\footnotetext{
Address for correspondence: Professor Cathy J Price Wellcome Trust Centre for Neuroimaging Institute of Neurology, UCL 12 , Queen Square London WC1N 3BG c.price@ fil.ion.ucl.ac.uk.

Author Contributions. C.J.P. designed and supervised the study. C.J.P and C.S recruited the participants. C.S., S.R. and G.J. collected the data. F.M.R., S.R., C.E., M.L.S and C.J.P analysed the data. S.R., M.S.C.T. and C.J.P wrote the manuscript and all authors edited the manuscript.
}

Author Information. Reprints and permissions information is available at www.nature.com/reprints. The authors declare no competing financial interests. 
neural markers for verbal and nonverbal (henceforth performance) IQ changes could in principle be dissociated. This would overcome two of the challenges faced by previous studies of inter-subject variability in IQ measures at a given time point: verbal and performance IQ are tightly correlated within individuals, so it has been hard to identify neural structures corresponding to $\mathrm{each}^{3,4}$; and there are many sources of between subject variance in brain structure (e.g. gender, age, size, handedness), that hide the differences of interest.

Our participants were thirty-three healthy and neurologically normal adolescents with a deliberately wide and heterogeneous mix of abilities (see Supplementary Information for details and the implications of our sampling for the generalisability of our conclusions). They were first tested in 2004 ("Time 1") when they were 12 to 16 years old (mean=14.1). Testing was repeated in 2007/2008 ("Time 2") when the same individuals were 15 to 20 years old (mean=17.7). See Table 1 for further details of the participants. During the intervening years, there were no testing sessions and participants and their parents had no knowledge that they would be invited back for further testing. On both occasions each participant had a structural brain scan using magnetic resonance imaging (MRI), and had their IQ measured using the Wechsler Intelligence Scale for Children (WISC-III) at Time 1 and the Wechsler Adult Intelligence Scale (WAIS-III) at Time 2 (see Supplementary Information for details). These two widely used age-appropriate assessments ${ }^{5}$ produce strongly correlated results at a given time point, consistent with them measuring highly similar constructs ${ }^{6}$. Scores on individual sub-tests are standardized against age-specific norms, and then grouped to produce separate measures of Verbal and Performance IQ (VIQ and PIQ), with the former encompassing those tests most related to verbal skills and the latter being more independent of verbal skills. Nevertheless, VIQ and PIQ scores are very significantly correlated with each other across participants: in our sample, the correlations between VIQ and PIQ were: $\mathrm{r}=0.51$ at Time 1 ; and $\mathrm{r}=0.55$ at Time 2 (in both cases $\mathrm{n}=33$; $\mathrm{p}<0.01$ ). Full Scale IQ (FSIQ) is the composite of these VIQ and PIQ skills and is regarded as the best measure of general intellectual capacity (the $\mathrm{g}$ factor) that has previously been shown to correlate with brain size and cortical thickness in a wide variety of frontal, parietal and temporal brain areas ${ }^{7-8}$.

The wide range of abilities in our sample was confirmed as follows: FSIQ ranged from 77 to 135 at Time 1 and 87 to 143 at Time 2, with averages of 112 and 113 at Times 1 and 2 respectively, and a tight correlation across testing points $(r=0.79 ; \mathrm{p}<0.001)$. Our interest was in the considerable variation observed between testing points at the individual level which ranged from -20 to +23 for VIQ, -18 to +17 for PIQ and -18 to +21 for FSIQ. Even if one adopts the extreme values of the published $90 \%$ confidence intervals on both occasions, $39 \%$ of the sample showed a clear change in VIQ, 21\% in PIQ, and 33\% in FSIQ. In terms of the overall distribution, $21 \%$ of our sample showed a shift of at least one population standard deviation (15) on the VIQ measure, and $18 \%$ on the PIQ measure. However, only one participant had a shift of this magnitude on both measures, and, in this case, one measure showed an increase and the other a decline. This pattern is reflected in the absence of a significant correlation between the change in VIQ and the change in PIQ. The independence of changes in these two measures allows us to investigate the effect of each without confounding influences from the other.

In order to test whether the observed IQ changes were meaningfully reflected in brain structure, they were correlated with changes in local brain structure. This within subject correlation eschews the many possible sources of between subject variance and may have sensitized our analysis to neural markers of VIQ and PIQ that have not previously been revealed. Given the distributed nature of brain regions associated with between subject differences in $\mathrm{FSIQ}^{7-9}$, regions of interest were not used in this analysis, and the results of 
the whole brain analysis were only considered to be significant at $p<0.05$ after family wise error correction in either height (peak signal at a single voxel) or extent (number of voxels that are significant at $\mathrm{p}<0.001)$.

Using a regression analysis, we looked at the brain changes associated with a change in VIQ, PIQ or FSIQ (see Methods Summary for details). The results (see Figure 1) showed that changes in VIQ were positively correlated with changes in grey matter density (and volume) in an area of the left motor cortex that is activated by the articulation of speech ${ }^{10}$. Conversely, changes in PIQ were positively correlated with grey matter density in the anterior cerebellum (lobule IV), which is associated with motor movements of the hand ${ }^{11-12}$. Post-hoc tests that correlated structural change with change on each of the nine VIQ and PIQ subtest scores that were common in the WISC and WAIS assessments found that the neural marker for VIQ indexed constructs that were shared by all VIQ measures, while the neural marker for PIQ indexed constructs that were common to three of the four PIQ measures (see Table 2). This indicates that our VIQ and PIQ markers indexed skills that were not specific to individual sub-tests. There were no other grey or white matter effects that reached significance in a whole brain structural analysis of VIQ, PIQ or FSIQ. See Supplementary Information for details of further post-hoc tests.

Our findings that changes in VIQ were related to a motor speech area and PIQ changes were related to a motor hand area are consistent with previous claims that cognitive intelligence is partly dependent on sensorimotor skills ${ }^{13-18}$. Using functional imaging in the same 33 participants performing a range of sensory, motor and language tasks, we confirmed that the left motor speech area identified in the VIQ structural analysis was more activated by articulation tasks (naming, reading, saying "1, 2, 3") than by semantic or perceptual tasks that required a finger press response (see Supplementary Information for details). In contrast, an area very close to the anterior cerebellum area identified in the PIQ structural analysis was more activated during tasks involving finger presses than during tasks involving articulation. Figure 2 shows these results at both the group and individual levels. The locations of the grey matter changes associated with VIQ and PIQ changes do not correspond to the anterior frontal and parietal regions associated with general intelligence $(\mathrm{g})^{7}$. It may therefore be the case that $\mathrm{g}$ remains relatively constant across age but changes in the ability to perform individual subtests depend on changes in sensorimotor skills. It is also notable that while completion of the sub-tests comprising verbal and performance measures must implicate a network of brain areas, only structural changes in regions associated with sensorimotor skills showed correlations with changes in VIQ and PIQ.

The changes in brain structure that correlated with changes in IQ allow us to explain some of the variance in terms of brain development. Specifically, $66 \%$ of the variance in Time 2 VIQ was accounted for by Time 1 VIQ, a further $20 \%$ was accounted for by the change in grey matter density in the left motor speech region, with the remaining $14 \%$ unaccounted for. Similarly, 35\% of the variance in Time 2 PIQ was accounted for by Time 1 PIQ, with $13 \%$ accounted for by the change in grey matter density in the anterior cerebellum, leaving $52 \%$ unaccounted for. Future studies may be able to account for more of the between subject variability by using a similar methodology with larger samples or other methodologies that measure structural or functional connectivity 8,19 .

These findings demonstrate considerable effects of brain plasticity in our sample during the teenage years, over and above normal development. By eschewing the many sources of between subject variance and controlling for global changes in brain structure, our within subject analysis has allowed us to dissociate brain regions where structure reflects individual differences in verbal or nonverbal performance, in a way that has proved difficult in previous studies using behavioural data from a single point in time. We have also shown that 
the changes observed over time in the IQ scores of teenagers cannot simply be measurement error, because they correlate with independently measured changes in brain structure in areas that are plausibly related to the verbal and nonverbal functions tested. Further studies are required to determine the generalisability of this finding: for example, is the same degree of plasticity present throughout life or are the adolescent years covered by this study special in this regard. In addition, future work could consider the causes of the identified changes in both intelligence and brain structure and how they impact on educational performance and employment prospects. Nevertheless, the implication of our finding is that an individual's strengths and weaknesses in skills relevant to education and employment are still emerging or changing in the teenage years.

\section{Methods Summary}

The study was approved by the joint ethics committee of the Institute of Neurology and the National Hospital for Neurology and Neurosurgery, London, UK. All structural and functional scans at Time 1 and Time 2 were acquired from the same Siemens 1.5T Sonata MRI scanner (Siemens Medical Systems, Erlangen, Germany). The structural images were acquired using a T1-weighted Modified Driven Equilibrium Fourier Transform sequence with 176 sagittal partitions and an image matrix of $256 \times 224$, yielding a final resolution of $1 \mathrm{~mm}^{3}$ [repetition time / echo time / inversion time $=12.24 \mathrm{~ms} / 3.56 \mathrm{~ms} / 530 \mathrm{~ms}$ ]. Preprocessing of 66 structural images (33 participants $\times 2$ time points) used SPM8 (http:// www.fil.ion.ucl.ac.uk/spm) with the DARTEL toolbox to segment and spatially normalize the brains into the same template; with and without modulation. Modulated images incorporate a measure of local brain volume while unmodulated images, used with proportional scaling to correct for global grey matter, provide a measure of regional grey matter density. Previous studies ${ }^{20-22}$ have shown the correlations between brain structure and cognitive ability are better detected by grey matter density. Co-ordinates for each voxel were converted to standard MNI space. Images were smoothed using an $8 \mathrm{~mm}$ isotropic Gaussian kernel at full width half maximum (FWHM). The relationship between change in IQ and change in brain structure was investigated by entering the appropriate pre-processed images (modulated or unmodulated grey or white matter) into within subjects paired t-tests, with change in IQ (VIQ, PIQ or FSIQ) and year of scan as covariates. The degree to which Time 2 IQ was predicted by changes in brain structure was investigated in a hierarchical regression analysis with Time 1 IQ entered before change in brain structure. Details of the functional imaging paradigm have been reported elsewhere ${ }^{23-25}$ and are summarised in Supplementary Information).

\section{Supplementary Material}

Refer to Web version on PubMed Central for supplementary material.

\section{Acknowledgments}

This work was funded by the Wellcome Trust. We thank Janice Glensman, Amanda Brennan, Alex Peters, Lorna Stewart, Kathy Pitcher and Dr Roy Rutherford for their help with data collection; and Will Penny for his advice on statistical analyses.

\section{References}

1. McCall RB. Childhood IQ's as predictors of adult educational and occupational status. Science. 1977; 197:482-483. [PubMed: 17783247]

2. Deary IJ, Whalley LJ, Lemmon H, Crawford JR, Starr JM. The stability of differences in mental ability from childhood to old age: follow-up of the 1932 Scottish Mental Survey. Intelligence. 2000; 28(1):49-55. 
3. Wilke M, Sohn J-H, Byars AW, Holland SK. Bright spots: correlations of gray matter volume with IQ in a normal pediatric population. NeuroImage. 2003; 20:202-215. [PubMed: 14527581]

4. Gong Q-Y, et al. Voxel_based morphometry and stereology provide convergent evidence of the importance of medial prefrontal cortex for fluid intelligence in healthy adults. NeuroImage. 2005; 25:1175-1186. [PubMed: 15850735]

5. Camara WJ, Nathan JS, Puente AE. Psychological test usage: implications in professional psychology. Professional Psychology: Research and Practice. 2000; 31(2):141-154.

6. Kaufman, A.; Lichtenberger, EO. Assessing Adolescent and Adult Intelligence. Wiley; New Jersey: 2006.

7. Haier RJ, Jung RE, Yeo RA, Head K, Alkire MT. Structural brain variation and general intelligence. NeuroImage. 2004; 23:425-433. [PubMed: 15325390]

8. Colom R, Karama S, Jung RE, Haier RJ. Human intelligence and brain networks. Dialogues Clin Neurosci. 2010; 12(4):489-501. [PubMed: 21319494]

9. Shaw P, et al. Intellectual ability and cortical development in children and adolescents. Nature. 2006; 440:676-679. [PubMed: 16572172]

10. Huang J, Carr TH, Cao Y. Comparing cortical activations for silent and overt speech using eventrelated fMRI. Hum. Brain Mapp. 2001; 15:39-53. [PubMed: 11747099]

11. Nitschke MF, Kleinschmidt A, Wessel K, Frahm J. Somatotopic motor representation in the human anterior cerebellum: a high-resolution functional MRI study. Brain. 1996; 119(3):1023-9. [PubMed: 8673479]

12. Stoodley CJ, Valerad EM, Schmahmann JD. An fMRI study of intra-individual functional topography in the human cerebellum. Behav. Neurol. 2010; 23:65-79. [PubMed: 20714062]

13. Diamond A. Close interrelation of motor development and cognitive development and of the cerebellum and prefrontal cortex. Child Devel. 2000; 71:44-56. [PubMed: 10836557]

14. Pangelinan MM, et al. Beyond age and gender: relationships between cortical and subcortical brain volume and cognitive-motor abilities in school-age children. NeuroImage. 2011; 54(4):3093-3100. [PubMed: 21078402]

15. Davis AS, Pass LA, Finch WH, Dean RS, Woodcock RW. The canonical relationship between sensory-motor functioning and cognitive processing in children with attention-deficit/hyperactivity disorder. Arch. Clin. Neuropsych. 2009; 24:273-286.

16. Davis EE, Pitchford NJ, Jaspan T, McArthur D, Walker D. Development of cognitive and motor function following cerebellar tumour injury sustained in early childhood. Cortex. 2010; 46:919932. [PubMed: 20338554]

17. Rosenbaum DA, Carlson RA, Gilmore RO. Acquisition of intellectual and perceptual-motor skills. Annu. Rev. Psych. 2001; 52:453-70.

18. Wassenberg R, et al. Relation between cognitive and motor performance in 5- to 6-year-old children: results from a large-scale cross-sectional study. Child Dev. 2005; 76:1092-1103. [PubMed: 16150004]

19. Jung RE, Haier RJ. The Parieto-Frontal Integration Theory (P-FIT) of intelligence: converging neuroimaging evidence. Behav. Brain Sci. 2007; 30(2):135-54. [PubMed: 17655784]

20. Eckert MA, et al. To modulate or not to modulate: differing results in uniquely shaped Williams syndrome brains. NeuroImage. 2006; 32(3):1001-7. [PubMed: 16806978]

21. Lee H, et al. Anatomical traces of vocabulary acquisition in the adolescent brain. J. Neurosci. 2007; 27(5):1184-9. [PubMed: 17267574]

22. Richardson FM, Thomas MS, Filippi R, Harth H, Price CJ. Contrasting effects of vocabulary knowledge on temporal and parietal brain structure across lifespan. J. Cogn. Neurosci. 2010; 22(5):943-54. [PubMed: 19366285]

23. Seghier ML, Fagan E, Price CJ. Functional subdivisions in the left angular gyrus where the semantic system meets and diverges from the default network. J Neurosci. 2010; 30(50):1680917. [PubMed: 21159952]

24. Seghier ML, Price CJ. Dissociating functional brain networks by decoding the between-subject variability. NeuroImage. 2009; 45(2):349-59. [PubMed: 19150501] 
25. Parker Jones O, et al. Where, when and why brain activation differs for bilinguals and monolinguals during picture naming and reading aloud. Cereb Cortex. 2011 

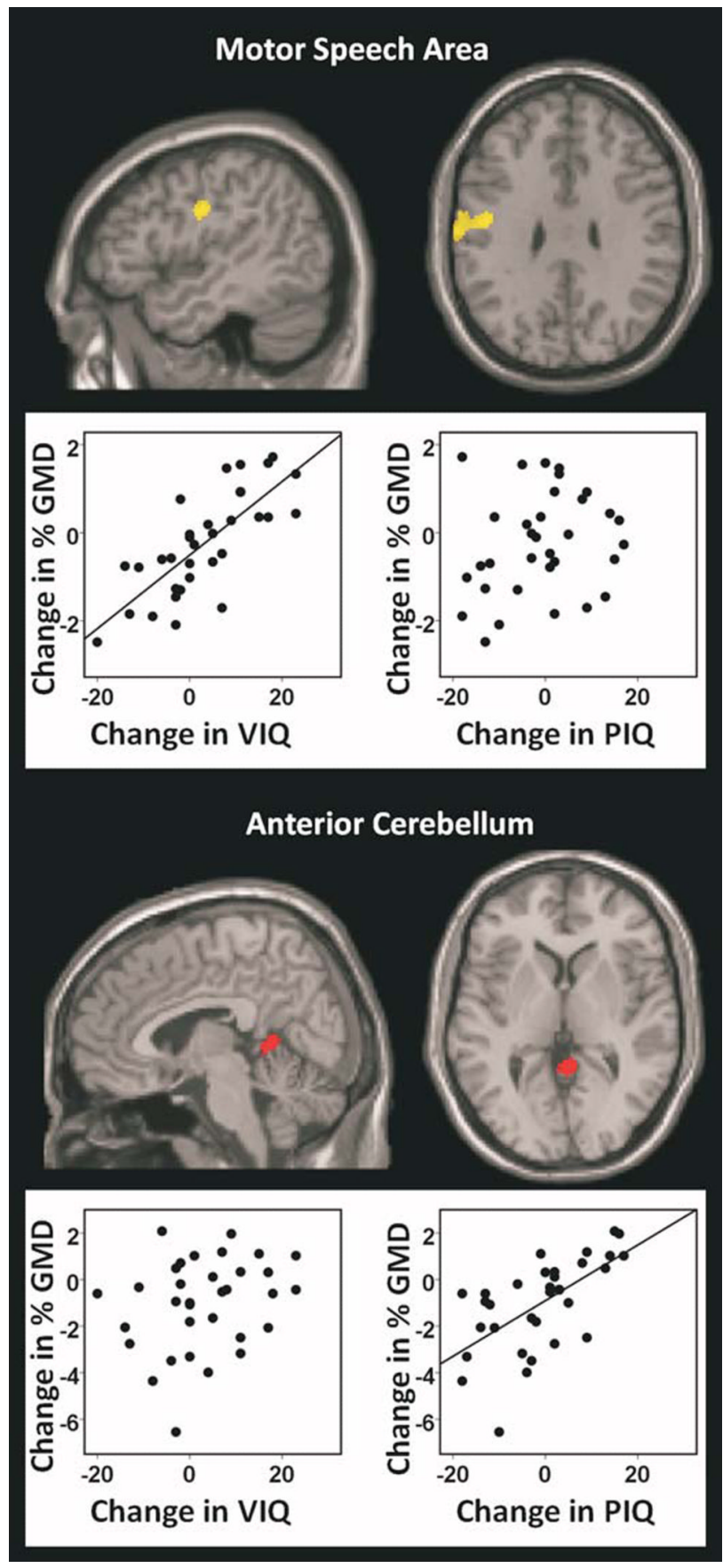

Figure 1.

Location of brain areas where grey matter changed with VIQ and PIQ. Top the correlation between change in grey matter density and change in VIQ (yellow) in the left motor speech area [peak in the left precentral gyrus at $x=-47, y=-9, z=+30$; with $Z$ score of 5.2 and 681 voxels at $\mathrm{p}<.001]$. The corresponding effect on volume was slightly less significant ( $Z$ score $=3.5 ; 118$ voxels at $\mathrm{p}<.001$ ). Bottom, the correlation between change in PIQ (red) and change in grey matter density in the anterior cerebellum [peak at $x=+6, y=-46 ; z=+3$; with $\mathrm{Z}$ score of 3.9 with 210 voxels at $\mathrm{p}<.001]$. Both effects were significant at $\mathrm{p}<0.05$ after FWE correction for multiple comparisons in extent based on the number of voxels in a cluster that survived $\mathrm{p}<0.001$ uncorrected. In addition, the VIQ effect was significant at $\mathrm{p}<0.05$ after 
FWE correction for multiple comparisons in height. The statistical threshold used in the Figure $(p<0.001)$ illustrates the extent of the effects. Plots show the change in grey matter density against the change in both VIQ and PIQ at the voxel with the highest Z score in the appropriate area. Changes in the motor speech area correlated with changes in VIQ but not changes in PIQ, while changes in the anterior cerebellum correlated with changes in PIQ but not changes in VIQ $(\mathrm{p}<.001) . \mathrm{n}=33$. GMD = grey matter density. 


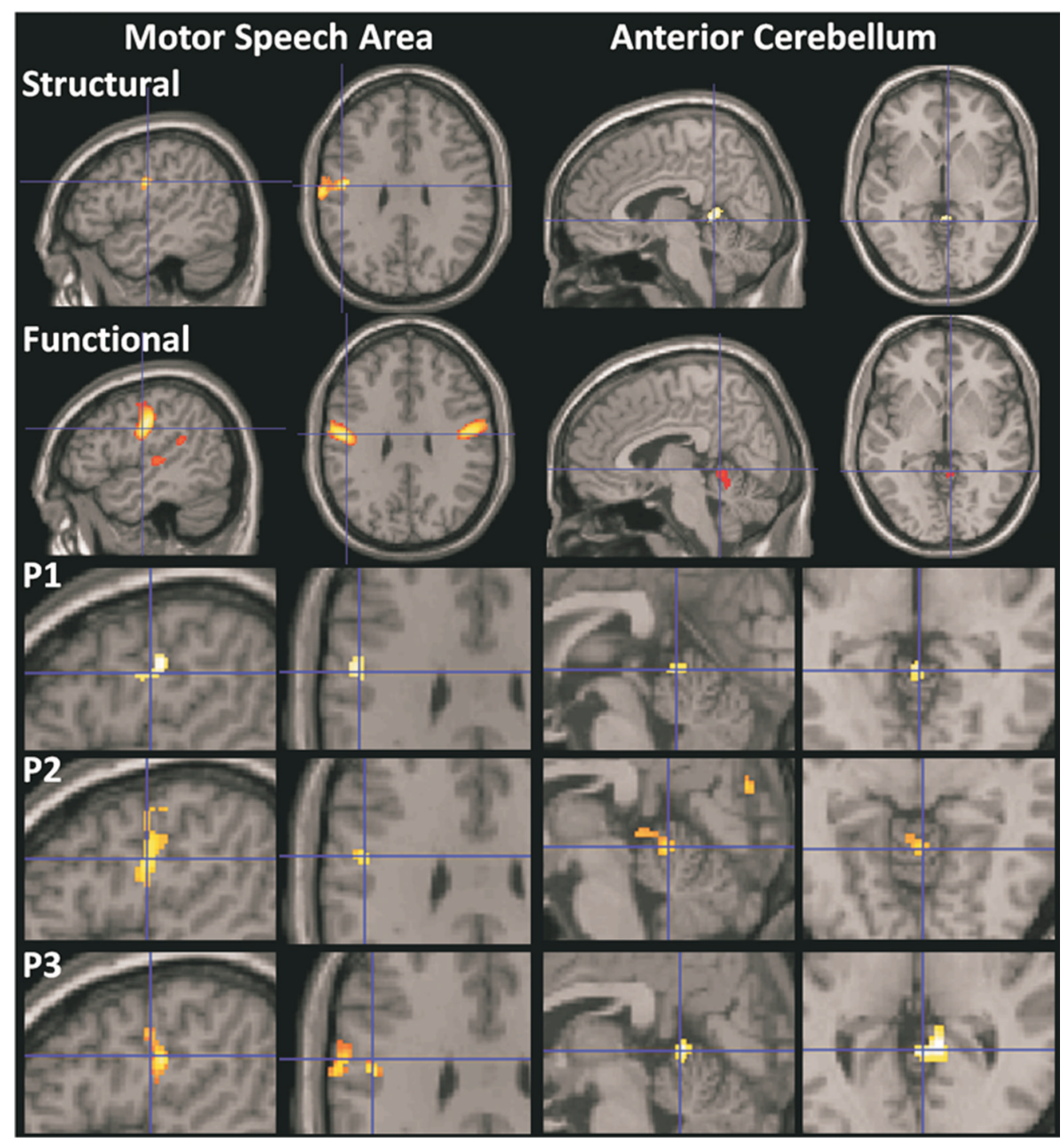

Figure 2.

Functional activations in the areas identified by the structural analysis The motor speech area was more activated by articulation tasks than by finger press tasks: $[\mathrm{x}=-48, \mathrm{y}=-10, \mathrm{z}=$ $+30 ; \mathrm{t}=14.7 ; \mathrm{p}<0.05 \mathrm{FWE}$ corrected for multiple comparisons across the whole brain], and corresponds to the area identified in the structural analysis for VIQ. These effects were consistently observed at the same coordinates for all individual subjects. In the three exemplar participants shown here (P1, P2, P3), the Z scores were 3.9, 3.5 and 3.0 respectively. The anterior cerebellum area was more activated during finger presses than articulation at both the Group level [peak at $\mathrm{x}=+6, \mathrm{y}=-48 ; \mathrm{z}=-4 ; \mathrm{Z}$ score $=3.7 ; 216$ voxels at $\mathrm{p}<.001$ corrected for multiple comparisons in extent] and individual level [P1: $\mathrm{x}=+12, \mathrm{y}=$ $-48, \mathrm{z}=+2 ; \mathrm{Z}$ score $=3.7 ; \mathrm{P} 2: \mathrm{x}=+6, \mathrm{y}=-50, \mathrm{z}=-6 ; \mathrm{Z}$ score $=3.3 ; \mathrm{P} 3: \mathrm{x}=+12, \mathrm{y}=-46, \mathrm{z}=+2$; $\mathrm{Z}$ score $=4.9$ ]. In all cases, the activation peaks were identified from whole brain analyses and the peak effects for the correlation with structure are illustrated with blue cross hairs in both the structural and functional results. This illustrates that the location of the structural effects is within the areas identified in the functional effects. 
Table 1

Participants' details

\begin{tabular}{|cccccc|}
\hline \multicolumn{2}{c}{ N = 33 (male 19) } & Age & FSIQ & VIQ & PIQ \\
Time 1 & Mean (SD) & $14.1(1.0)$ & $112(13.9)$ & $113(15.1)$ & $108(12.3)$ \\
& Min/max & $12.6 / 16.5$ & $77 / 135$ & $84 / 139$ & $74 / 137$ \\
\hline \multirow{2}{*}{ Time 2 } & Mean (SD) & $17.7(1.0)$ & $113(14.0)$ & $116(18.0)$ & $107(9.6)$ \\
& Min/max & $15.9 / 20.2$ & $87 / 143$ & $90 / 150$ & $83 / 124$ \\
\hline \multirow{2}{*}{ Correlation 1} & $\mathbf{r}$ & & $.792 * *$ & $.809 * *$ & $.589 * *$ \\
\hline Change & Mean (SD) & $3.5(0.2)$ & $+1.0(9.0)$ & $+3.0(10.6)$ & $-1.0(10.2)$ \\
(Time 2 -Time 1) & Min/max & $3.3 / 3.9$ & $-18 /+21$ & $-20 /+23$ & $-18 /+17$ \\
\hline
\end{tabular}

${ }^{1}$ Correlation coefficient between Time 1 and Time 2 scores

SD standard deviation

*** significant at $\mathrm{p}<.01$ level 
Table 2

Correlation between change in grey matter density and change in sub-test score

\begin{tabular}{|l|l|c|c|}
\hline \multicolumn{1}{|c|}{ Correlation coefficients (r) } & $\begin{array}{c}\text { Motor } \\
\text { speech area }\end{array}$ & $\begin{array}{c}\text { Anterior } \\
\text { cerebellum }\end{array}$ \\
\hline \multirow{4}{*}{ Verbal tests } & Vocabulary & $.284^{\dagger}$ & .142 \\
\cline { 2 - 4 } & Similarities & $.438^{* *}$ & -.021 \\
\cline { 2 - 4 } & Arithmetic & $.477^{* *}$ & $.304^{*}$ \\
\cline { 2 - 4 } & Information & $.314^{*}$ & .185 \\
\cline { 2 - 4 } & Comprehension & $.541^{* *}$ & .183 \\
\hline \multirow{4}{*}{ Nonverbal tests } & Picture Completion & .038 & $.363^{*}$ \\
\cline { 2 - 4 } & Digit Symbol Coding & .003 & -.028 \\
\cline { 2 - 4 } & Block Design & .000 & $.306^{*}$ \\
\cline { 2 - 4 } & Picture Arrangement & .126 & $.437^{* * *}$ \\
\hline \multirow{3}{*}{ Significant (one-tailed) at p<.01 level } & & \\
\multirow{2}{*}{$\begin{array}{l}* \\
\text { Significant (one-tailed) at p<.05 level } \\
\text { Trend (one-tailed) at p=.0545 }\end{array}$}
\end{tabular}

Correlations calculated using changes in scaled (i.e. age-adjusted) scores in the various sub-tests which were common to both the WISC and the WAIS. The change in grey matter density in the motor speech region correlated significantly with changes in scores in four of the five verbal subtests and there was a near-significant trend with the fifth, but it did not correlate significantly with changes in scores in any of the four tests that comprise PIQ. Conversely, the change in grey matter density in the anterior cerebellum correlated significantly with changes in scores in three of the four tests that comprise PIQ (the exception being Digit Symbol Coding which has a particular loading on processing speed), but only correlated with changes in scores in one of the verbal tests (Arithmetic, which probably has the smallest verbal component of the verbal tasks). 